\title{
EXPERIMENTAL EVIDENCE OF IMPROVED THERMOELECTRIC PROPERTIES AT 300K IN Si/Ge SUPERLATTICE STRUCTURES
}

R. Venkatasubramanian, (A. C. Pezzi)

April1997

DISTRISUTION OF THIS DCCUMENT IS UNLMTEED $p^{h}$

\section{MASTER}

\section{NOTICE}

This report was prepared as an account of work sponsored by the United States Government. Neither the United States, nor the United States Department of Energy, nor any of their employees, nor any of their contractors, subcontractors, or their employees, makes any warranty, express or implied, or assumes any legal liability or responsibility for the accuracy, completeness or usefulness of any information, apparatus, product or process disclosed, or represents that its use would not infringe privately owned rights.

Operated for the U. S. Department of Energy

by KAPL, Inc. a Lockheed Martin company 


\section{DISCLAIMER}

This report was prepared as an account of work sponsored by an agency of the United States Goverament. Neither the United States Government nor any agency thereof, nor any of their employees, makes any warranty, express or implied, or assumes any legal liability or responsibility for the accuracy, completeness, or usefulness of any information, apparatus, product, or process diselosed or represents that its use would not infringe privately owned rights. Refereace berein to any specific commercial product, process, or service by trade name, trademart manufacturer, or otherwise does not necessarily constitute or imply its endorsement, recommendation, or favoring by the United States Governmeat or any ageacy thereof. The views and opinions of authors expresed hercin do not necessarily state or reflect those of the United States Government or any agency thereof. 


\section{DISCLAIMER}

Portions of this document may be illegible in electronic image products. Images are produced from the best available original document. 


\title{
Experimental Evidence of Improved Thermoelectric Properties at 300K in Si/Ge Superlattice Structures
}

\author{
R. Venkatasubramanian, T. Colpitts, E. Watko, and D. Malta \\ Research Triangle Institute, Research Triangle Park, NC 27709, USA.
}

\section{Abstract}

We have found that it may be possible to obtain significant enhancement in $\mathrm{ZT}$ at $300 \mathrm{~K}$, over conventional bulk $\mathrm{SiGe}$ alloys, through the use of $\mathrm{Si} / \mathrm{Ge}$ Superlattice (SL) structures. The Seebeck coefficient in Si/Ge SL structures was observed to increase rapidly with decreasing SL period with no loss of electrical conductivity. The carrier mobilities in Si/Ge SLs were higher than in a comparable thin-film SiGe alloy. The best power factor of the short-period Si/Ge SLs is 112.2 $\mu \mathrm{W} / \mathrm{K}^{2} \mathrm{~cm}$, over five-fold better than state-of-the-art n-type, bulk SiGe alloys. Approximately a two to four-fold reduction in thermal conductivity in short-period SL structures, compared to bulk $\mathrm{SiGe}$ alloy, was observed. We estimate at least a factor of five improvement over current state-of-the-art $\mathrm{SiGe}$ alloys, in several $\mathrm{Si} / \mathrm{Ge} \mathrm{SL}$ samples with periodicity of $\sim 45$ to $75 \AA$. The results of this study are promising, but tentative due to the possible effects of substrate and the developmental nature of the thermoelectric property measurements.

\section{Superlattice Structures for ZT Enhancement}

We discuss experimental work on a new class of structurally-engineered, high-performance, thin-film Si/Ge superlattice (SL) thermoelectric materials with a significantly enhanced figure-of-merit (ZT) at $300 \mathrm{~K}$ compared to those obtainable with present-day bulk $\mathrm{SiGe}$ alloy thermoelectric materials for improving the efficiency of thermoelectric power generation devices.

Whall and Parker [1] in 1987 suggested the possible enhancement in ZT of SiGe-thermoelectric materials by using SL structures, through carrier confinement in quantum wells to obtain an enhancement in Seebeck coefficient and by modulation doping to obtain conductivity enhancement. Recently [2] various approaches to obtain a significant breakthrough in the performance of thermoelectric materials have been considered. Thin-film approaches using SL structures to enhance power-factor $[3,4]$ and to decrease thermal conductivity without appreciable reduction in electrical conductivity [5] have been proposed. Elsner et. al of $\mathrm{Hi}-\mathrm{Z}$ Technology, in cooperation with the Naval Research Laboratory, reported on some significant enhancement of thermoelectric properties of $\mathrm{Si} \mathrm{Si}_{0.8} \mathrm{Ge}_{0.2}$ superlattice structures grown on conducting $\mathrm{Si}$ substrates [6]. The conducting nature of the $\mathrm{Si}$ substrate, especially when it is lightly-doped (therefore has a high Seebeck coefficient) and is of the same polarity as the epitaxial film that is being investigated, makes the interpretation of properties of superlattices very difficult. Further, in this study on $\mathrm{Si} / \mathrm{Si}_{0.8} \mathrm{Ge}_{0.2}$ SL structures, superlattice dimensional-related quantification of thermoelectric properties were not reported.
Such a quantification is important to distinguish that the improved properties are indeed from a superlattice approach, rather than arising purely from an epitaxial deposition process or other artifacts such as conduction from substrates. The purpose of this study is to report on the enhancement of thermoelectric properties of Si/Ge SL structures compared to bulk $\mathrm{SiGe}$ alloys, considered for use in power generation applications.

\section{Experimental}

All the Si/Ge SL structures and SiGe alloy thin-films reported in this study were intentionally doped n-type. The use of semi-insulating GaAs substrates is ideal for obtaining the resistivity of the $\mathrm{Si} / \mathrm{Ge}$ epitaxial layers by the van der Pauw Hall method and the determination of the in-plane Seebeck coefficients of the epi layers, as discussed below. Note that the SL period is defined as the sum total thickness of one cycle of $\mathrm{Si}$ and $\mathrm{Ge}$ layer; typically both these $\mathrm{Ge}$ and $\mathrm{Si}$ layer thicknesses are kept nominally equal through this study, unless specified. Shown in Figure 1 is the scanning electron microscopy cross-section (XSEM) view of $(\sim 4.5 \mu \mathrm{m}) \mathrm{Si} / \mathrm{Ge} \mathrm{SL}$ structures. We have also grown Si/Ge SL structures with a periodicity of $\sim 100 \AA$ and as thick as $\sim 10 \mu \mathrm{m}$; with the total layer thickness including the buffer of about $11 \mu \mathrm{m}$.

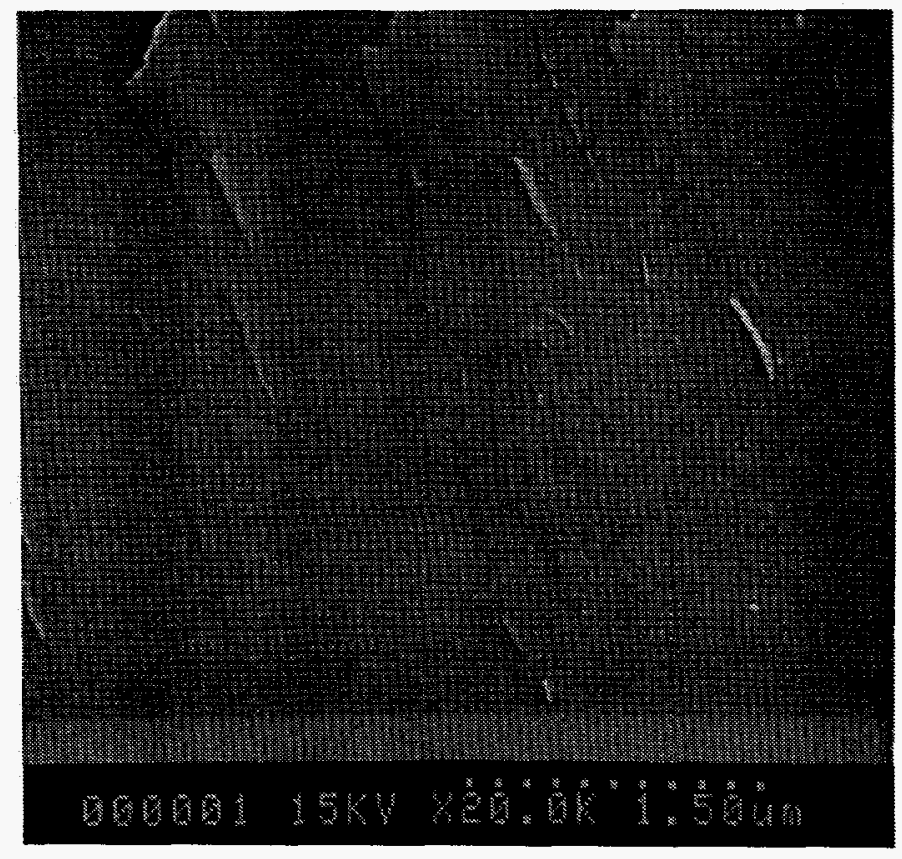

Figure 1. XSEM view of a 4.5-micron-thick, $100 \AA$-period $\mathrm{Si} / \mathrm{Ge}$ SL structure.

In extending the growth of $\mathrm{Si} / \mathrm{Ge}$ superlattice structures to shorter periods below $\sim 100 \AA$, we had to develop an alternate SEM technique denoted as angle-lapped scanning electron 
microscopy (ALSEM) to characterize the SL structures as the typical resolution limit of the SEM apparatus is $\sim 100 \AA$. Here, we investigated the approach of angle-lapping a crosssectioned short-period SL sample at a known, small angle such as $\sim 5^{\circ}$ and then examine the sample by SEM in a top-view. This was found to be adequate in this study to confirm the nominal calibration (based on individual growth rates of the $\mathrm{Si}$ and Ge layers) of short-period SL structures. The ALSEM technique was first applied to known large-period Si/Ge SL structures to confirm the accuracy of the determination of the SL period by ALSEM with that determined by conventional XSEM. Once the accuracy of the ALSEM technique was ensured, we applied it to short-period SL structures. Shown in Figure $2(\mathrm{a}, \mathrm{b})$ are the conventional XSEM and ALSEM views on a $35 \AA / 35 \AA \mathrm{Si} / \mathrm{Ge}$ SL structure, clearly indicating the usefulness of the ALSEM technique in the structural characterization of short-period SL structures.

\section{PROPERTIES OF Si/Ge SL STRUCTURES}

\section{Electron Mobility Dependence on SL Period}

Shown in Figure 3 is the observed variation of electron mobility (determined by Hall-effect) with the SL period. We have shown this dependence for a narrow range of free-carrier or impurity dopant levels, as we believe this reduces the effect of varying impurity scattering on electron mobility and thereby isolates the effect of the SL period on electron mobility. We clearly observe that the electron mobility improves with smaller period in the Si/Ge SL structures, for $\mathrm{n} \sim 3 \pm 1.3 \mathrm{E} 18 \mathrm{~cm}^{-3}$.

This can be understood from the $\mathrm{Si} / \mathrm{Ge} \mathrm{SL}$ heterostructure band diagram shown in Figure 4. The Si/Ge heterostructure band diagram (for a SL period $\cong 50 \AA$ ) indicated here is for ideal conditions, where the conduction band offsets are deduced from the electron affinity differences between $\mathrm{Ge}$ and $\mathrm{Si}$ and ignores the possible increase in bandgap of the $\mathrm{Si}$ layer from uniaxial expansion along the $<100>$ growth direction. Also, the energies of the $n=1$ and $\mathrm{n}=2$ quantum levels indicated in the Ge layer are simple estimations ignoring the most-likely case of electrons not being totally confined in the Ge layer, but also getting distributed in the conduction band of Si. For the n-type doped $\mathrm{Si} / \mathrm{Ge}$ materials, for doping levels such as $>3 \mathrm{E} 18 \mathrm{~cm}^{-3}$, the impurity scattering of carriers is expected to dominantly affect the mobility at $300 \mathrm{~K}$. The general dependence of time constants associated with impurity scattering and acoustic scattering of electrons in the conduction band of $\mathrm{Ge}$, as a function of energy separation from the conduction band edge of $\mathrm{Ge}$, is shown in Figure 4 (b). For the electrons in the Ge layer, as the $S L$ period decreases, the impurity scattering time constant increases and therefore the electron mobility increases.

A similar behavior of increasing electron mobility with shorter SL period is also observed for $\mathrm{n} 1.0 \pm 0.1 \mathrm{E} 19 \mathrm{~cm}^{-3}$ in Figure. 3 , till $\sim 100 \AA$, below which we see that the mobility actually falls with smaller periods. This is also true in the case of $\mathrm{n} \sim 1.5 \pm 0.2 \mathrm{E} 19 \mathrm{~cm}^{-3}$, for SL period $<75 \AA$. This fall in mobility is important, as the heavier doping levels and shorter SL periods tend to offer higher ZT values (in spite of this degradation in mobility) as discussed later. Thus, an understanding of this behavior and a possible solution to the fall in mobility can help to achieve even higher ZT values. In any case, it is important to note that for similar free-carrier levels, the mobility of all the Si/Ge SL structures are significantly higher than that of a comparably doped SiGe alloy epitaxial film, as indicated in Figure 3. The improved mobility is attributed to avoiding alloy scattering and minimizing impurity scattering in Si/Ge SL structures.

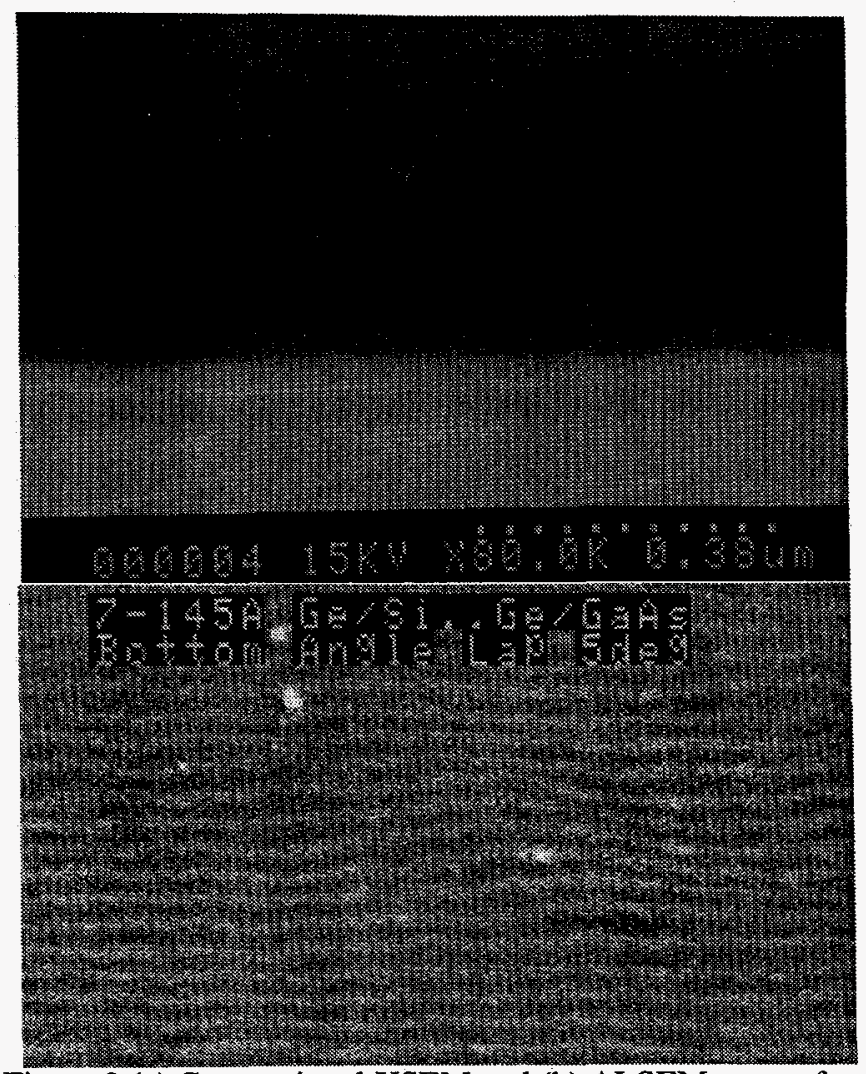

Figure 2 (a) Conventional XSEM and (b) ALSEM vews of a $35 \AA / 35 \AA \mathrm{Si} / \mathrm{Ge} \mathrm{SL}$ structure.

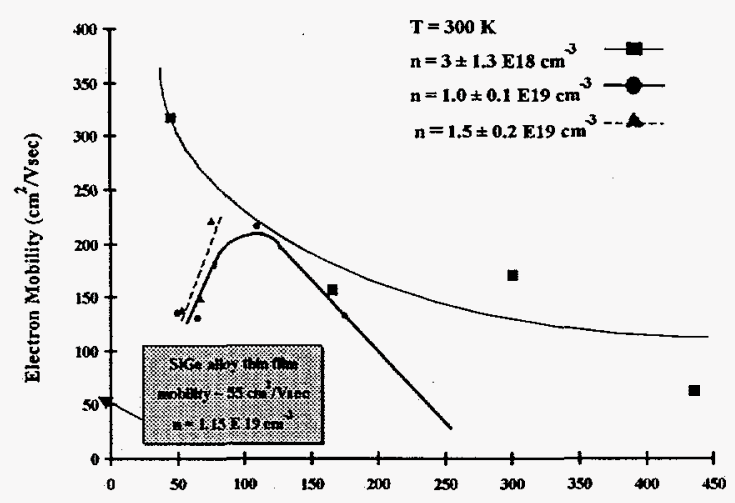

SL, Period $(\dot{A})$

Figure 3. Variation of electron mobility with SL period for a range of free-carrier levels. 
(a)

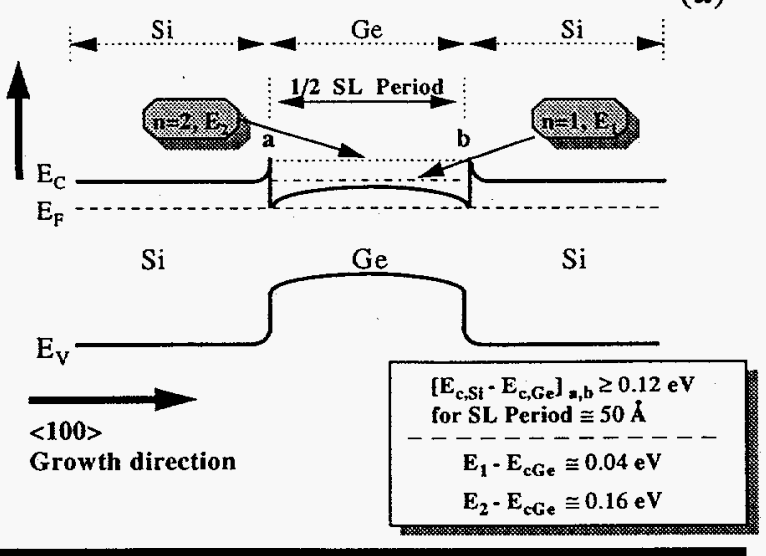

(b)

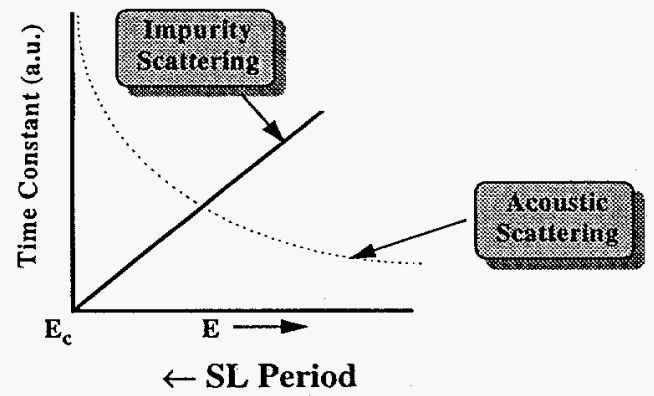

Figure 4 (a) Heterostructure band diagram at the Si/Ge SL interface and (b) the nature of time constants associated with impurity scattering and acoustic scattering of electrons in the conduction band of Ge.

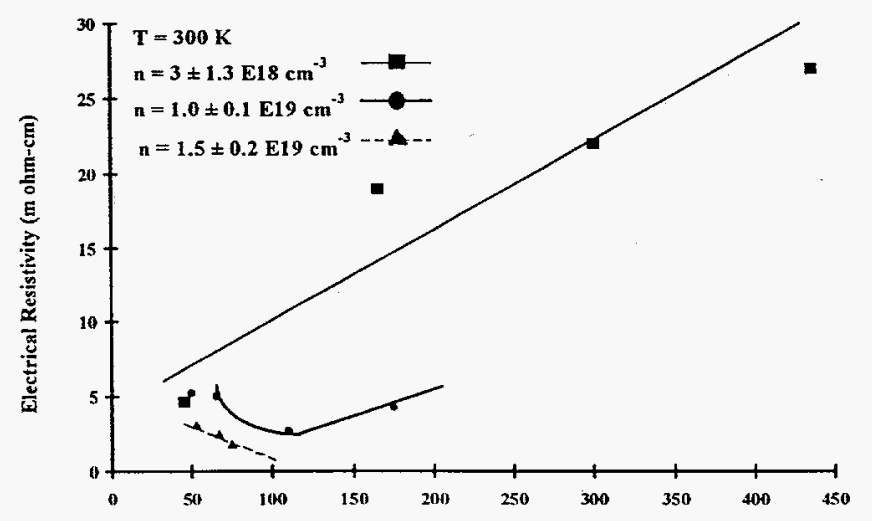

SL Period (A)

Figure 5 Variation of electrical resistivity with SL period for a range of free-carrier levels.

The electrical resistivity of $\mathrm{Si} / \mathrm{Ge} \mathrm{SL}$ structures, as a function of SL period free-carrier concentration, is shown in Figure 5. For $\mathrm{n}=3 \pm 1.3 \mathrm{E} 18 \mathrm{~cm}^{-3}$, we can observe that the electrical resistivity continuously falls with a decrease in the SL period, a result of improved electron mobility with shorter SL period. For $\mathrm{n}=1.0 \pm 0.1 \mathrm{E} 19 \mathrm{~cm}^{-3}$, the electrical resistivity initially falls with the SL period and then apparently increases with shorter periods, mimicking the behavior of electron mobility. For SL periods with $1.5 \pm 0.2 \mathrm{E} 19 \mathrm{~cm}^{-3}$, we clearly see an increase in electrical resistivity with shorter SL periods.

\section{Seebeck Coefficients in Si/Ge SL Structures}

Shown in Figure 6 is the variation of the in-plane Seebeck coefficient in Si/Ge SL structures as a function of SL period and carrier concentration levels. We note here that considerable differences remain in the measurement of Seebeck coefficients in various laboratories, although the general trends are similar. For all the free-carrier levels, we observed that the Seebeck coefficient does increase with a shorter SL period. This is similar to the observation of the enhancement of the Seebeck coefficient in $\mathrm{PbTe} / \mathrm{PbEuTe}$ quantum-wells [7] and $\mathrm{Bi}_{2} \mathrm{Te}_{3} / \mathrm{Sb}_{2} \mathrm{Te}_{3}$ superlattices [8]. Here in the $\mathrm{Si} / \mathrm{Ge} \mathrm{SL}$ structures, we see a rather sharper dependence of the Seebeck coefficient with the SL period for nearly all carrier levels. We see a near $80 \%$ increase in the Seebeck coefficient in going from a SL period of $300 \AA$ to about $45 \AA$, for a carrier concentration of $3 \pm 1.3 \mathrm{E} 18 \mathrm{~cm}^{-3}$; a $200 \%$ increase is seen going from SL period of $\sim 175 \AA$ to $50 \AA$ for $\mathrm{n}=1.0 \pm 0.1 \mathrm{E} 19 \mathrm{~cm}^{-3}$ and a similar rate of increase in the Seebeck coefficient with the SL period for $\mathrm{n}=1.5 \pm 0.2 \mathrm{E} 19 \mathrm{~cm}^{-3}$.

It is worth noting that for a SL period of $\sim 50 \AA$, the Seebeck coefficient of the $\mathrm{Si} / \mathrm{Ge} \mathrm{SL}$ structures is nearly independent of the carrier concentration, including some of the data on stacked Si/Ge SL structures as shown in Table 1. This is in contrast to the well-behaved higher Seebeck coefficient with lower free-carrier levels in bulk SiGe alloys. This can be understood from the fact that the Seebeck coefficient $(\alpha)$ is the ratio of the first moment of electrical conductivity $(\sigma)$ with respect to energy (E) to the zero moment of $\sigma$, given by the expression:

$$
\alpha=-\frac{\mathbf{k}}{\mathrm{e}}\left(\frac{\int_{\mathrm{Ec}}^{\infty} \mathrm{E} \sigma(\mathrm{E}) \mathrm{dE}}{\int \sigma(\mathrm{E}) \mathrm{dE}}-\mathrm{E}_{\mathrm{f}}\right)
$$

As seen from the heterostructure band diagram of this $\mathrm{Si} / \mathrm{Ge} \mathrm{SL}$ in Fig. 4 (a), nearly all the electrons in the Si/Ge SL structures are expected to be in the conduction band of $\mathrm{Ge}$ at a constant energy from the conduction band edge of $\mathrm{Ge}$. Further, if we assume all the electrons are in the first $(n=1)$ level, the integral term in the above expression becomes independent of $\mathrm{E}$ and the Seebeck coefficient is nearly independent of the conductivity $\sigma$. Then, for a given SL period such as $50 \AA$, the only variation in the Seebeck coefficient with carrier concentration arises from the position of the Fermi level $\left(\mathrm{E}_{\mathrm{f}}\right)$ with respect to the conduction band of Ge. At these doping levels, the Fermi level is expected to be nearly pinned at the donor ionization energy, in this case that of As, which is expected to be relatively constant with respect to the conduction band of Ge. In any case, the most likely quantum-confinement of electrons in $G e$ even if weak, apparently increases the Seebeck coefficient in the shortperiod (50 to $75 \AA$ ) Si/Ge SL structures, by as much as $68 \%$ 
over the value obtained in a comparably doped epitaxial SiGe alloy film.

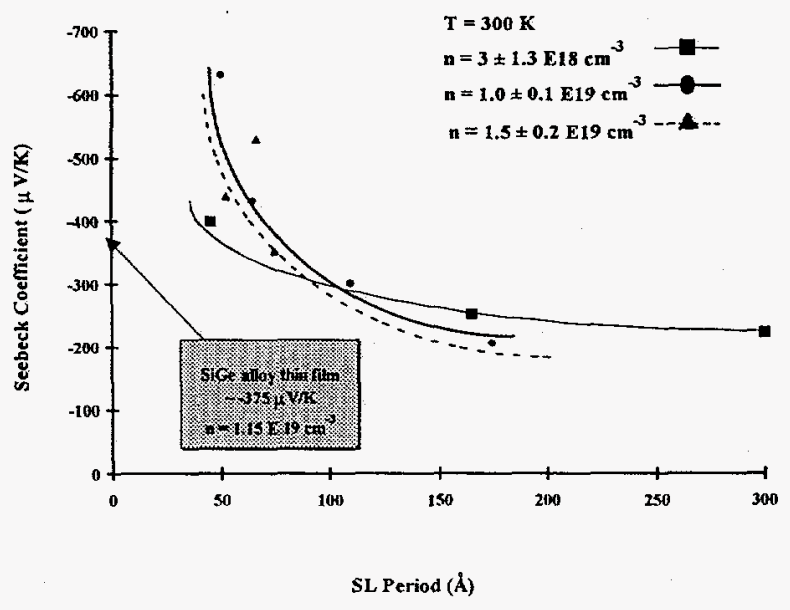

Figure 6 Variation of Seebeck coefficient with SL period for a range of free-carrier levels.

Table 1 Seebeck Coefficient in Si/Ge SL Structures and its Near Independence on Carrier Concentration

\begin{tabular}{|cccc|}
\hline $\begin{array}{c}\text { Sample } \\
\#\end{array}$ & $\begin{array}{c}\text { SL } \\
\text { Period } \\
(\AA)\end{array}$ & $\begin{array}{c}\text { Carrier } \\
\text { Concentration } \\
\text { n(cm } \mathbf{3})\end{array}$ & $\begin{array}{c}\text { Seebeck } \\
\text { Coefficient } \\
(\mu \mathbf{V} / \mathbf{K})\end{array}$ \\
\hline \hline $7-150$ & 45 & $0.4 \mathrm{E} 19$ & -400.7 \\
$7-153$ & 50 & $0.9 \mathrm{E} 19$ & -631.0 \\
$7-196$ & 53 & $1.5 \mathrm{E} 19$ & -438.3 \\
\hline
\end{tabular}

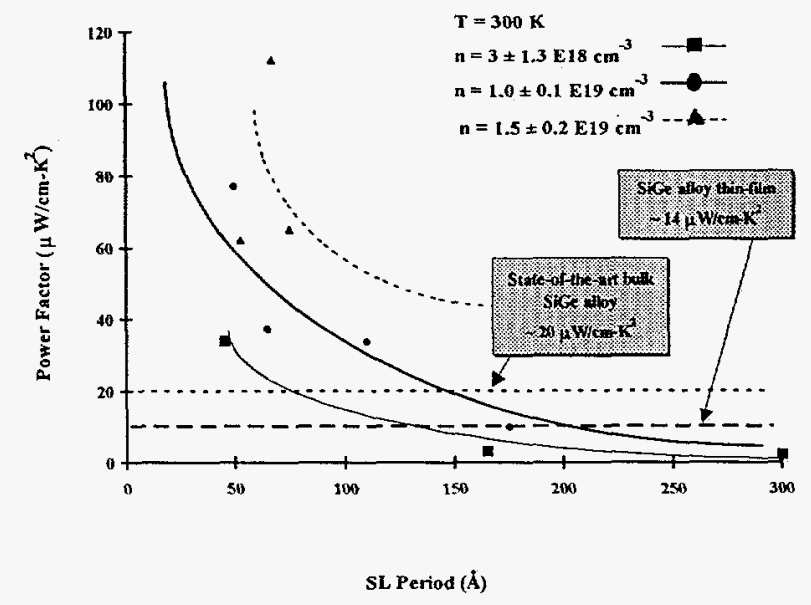

Figure $7 \quad$ Variation of power factor with SL period for a range of free carrier levels.

\section{Power Factor Improvement in Si/Ge SL Structures}

The combination of lower electrical resistivity from improved electron mobilities with short-period $(<150 \AA) \mathrm{Si} / \mathrm{Ge}$ SL structures and the improved Seebeck coefficient for shortperiod $(<75 \AA)$ Si/Ge SL structures, all lead to a significant improvement in power factors of the SL structures with smaller periods. This is indicated in Figure 7 . We can note from this data, in the free-carrier level range of $3 \mathrm{E} 18$ to $1.5 \mathrm{E} 19 \mathrm{~cm}^{-3}$, that there is a significant improvement in the power factor even with shorter-periods at higher free-carrier levels. This is to be expected from the aforementioned near independence of the Seebeck coefficient on the free-carrier level and the less-than-linear degradation in electron mobility with higher free-carrier levels. Shown in Figure 7 is the power factor typically obtained on state-of-the-art SiGe bulk alloy, approximately $\sim 20 \mu \mathrm{W} / \mathrm{cm}-\mathrm{K}^{2}$. The power factor measured on a SiGe alloy thin-film, for reference, was $-14 \mu \mathrm{W} / \mathrm{cm}-\mathrm{K}^{2}$. Thus the best measured power factor in Si/Ge SL structures is a factor of 5.6 better than the state-of-the-art bulk SiGe alloy and as much as a factor of 8.0 better than a SiGe alloy film.

\section{Thermal Conductivity in Si/Ge SL Structures}

Shown in Figure 8 is the distribution of thermal conductivity $(\mathrm{K})$ determined by the 3- $\omega$ method [9] on $\mathrm{Si} / \mathrm{Ge}$ SL structures. The variation of $\mathrm{K}$ with SL period is grouped as a function of free-carrier levels as we believe that the electronic contribution to thermal conductivity could play an important role, especially if the lattice thermal conductivity is reduced by the use of SL structures.

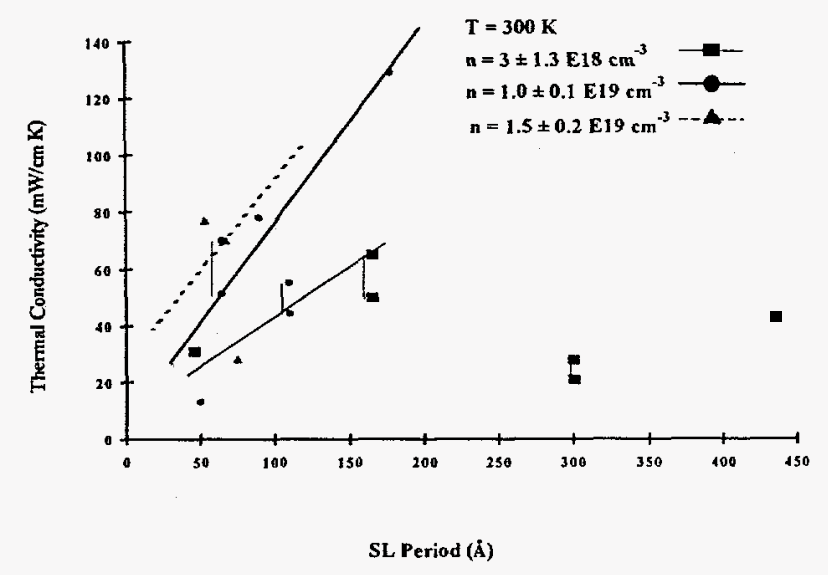

Figure 8 Variation of thermal conductivity with SL period for a range of free carrier levels.

This is evident in the data shown in Table 2, where we have compared the thermal conductivity of $\mathrm{Si} / \mathrm{Ge} \mathrm{SL}$ structures for a set of constant SL periods, but varying electron concentration. It is interesting to note from this data that for a near constant SL period, the thermal conductivity increases with electron concentration. More importantly, the rate of increase in thermal conductivity with carrier concentration is higher at a smaller SL period compared to SL structures with larger periods. This data is consistent with reduced lattice thermal conductivity contribution to the overall thermal conductivity with shorter SL periods. The same effect is observed for comparable electron concentration and, hence, comparable electronic contribution to thermal conductivity, quantitatively shown in Figure 8. We have shown in this figure that, under the limiting condition where lattice thermal conductivity is essentially negligible with SL period 
approaching very small values, the intercepts are a function of the carrier levels.

Table 2 Dependence of Thermal Conductivity of Si/Ge SL structures on Free-Carrier Concentration

\begin{tabular}{|cccc|}
\hline $\begin{array}{c}\text { Sample } \\
\#\end{array}$ & $\begin{array}{c}\text { SL } \\
\text { Period } \\
(\AA)\end{array}$ & $\begin{array}{c}\text { Free Carrier } \\
\text { Concentration } \\
\left(\mathbf{c m}^{-3}\right)\end{array}$ & $\begin{array}{c}\text { Thermal } \\
\text { Conductivity } \\
(\text { W/cmK })\end{array}$ \\
\hline \hline $7-144$ & 165 & $2.1 \mathrm{E} 18$ & 0.057 \\
$7-175$ & 178 & $8.8 \mathrm{E} 18$ & 0.129 \\
$7-154$ & 65 & $9.7 \mathrm{E} 18$ & 0.051 \\
$7-195$ & 67 & $1.66 \mathrm{E} 19$ & 0.07 \\
$7-150$ & 45 & $4.2 \mathrm{E} 18$ & 0.031 \\
$7-196$ & 53 & $1.5 \mathrm{E} 19$ & 0.077 \\
\hline
\end{tabular}

\section{ZT Values in Si/Ge SL Structures}

The Seebeck coefficient and the electrical conductivity of the Si/Ge SL structures are determined in the plane of the SL structures. The 3- $\omega$ method essentially gives the thermal conductivity of $\mathrm{Si} / \mathrm{Ge} \mathrm{SL}$ structures perpendicular to the SL interfaces. However, considering the correlation between the in-plane measured carrier levels and the perpendicular thermal conductivity values, perhaps only a small amount of anisotropy exists in the thermal properties of $\mathrm{Si} / \mathrm{Ge} \mathrm{SL}$ structures. Also, phonon mean free paths in pure $\mathrm{Si}$ and pure $\mathrm{Ge}$ are $76 \AA$ and $105 \AA$, respectively, and they are comparable to the SL dimension (a half of SL period) considered in this study. Hence the $\mathrm{ZT}$ of $\mathrm{Si} / \mathrm{Ge} \mathrm{SL}$ structures at $300 \mathrm{~K}$ are estimated assuming that the thermal conductivity of the SL structures in the plane of SL interfaces is about the same as that measured perpendicular to the SL interfaces. Such an estimated ZT at 300K is shown in Figure 9, as a function of SL period, for various free-carrier levels. The improvement in ZT offered by short-period SL structures, with a periodicity of 50 to $75 \AA$, at all free-carrier levels, is clear. The best ZT of $\sim 1.78$ was obtained. However, several samples with $\mathrm{ZT}$ in the range of 0.33 to 0.7 have been obtained.

We indicate a ZT of $~ 0.06$ obtained on a comparably doped thin-film SiGe alloy. Thus the thin-film, short-period $\mathrm{Si} / \mathrm{Ge}$ SL structures apparently offer a minimum of a ten-fold ZT improvement over thin-film SiGe alloys. A ZT of $\sim 0.12$ for state-of-the-art bulk SiGe alloy is indicated. Thus the thin-film, short-period Si/Ge SL structures apparently offer a minimum of a five-fold ZT improvement over bulk SiGe alloys.

\section{Conclusions and Summary}

Under certain doping levels, the electron mobility of the $\mathrm{Si} / \mathrm{Ge} \mathrm{SL}$ structures were found to increase significantly with smaller superlattice periods. This provides some evidence that the SL approach may help to avoid alloy scattering and minimize impurity scattering of carriers, thereby offering improved electrical conductivities. The Seebeck coefficients of the $\mathrm{Si} / \mathrm{Ge} \mathrm{SL}$ structures were found to increase rather significantly with smaller periods as well. For periods less than $\sim 75 \AA$, the SL structures had a higher (factor of 1.5 to 1.7 )
Seebeck coefficient than comparably-doped SiGe alloy film. These contributed to achieving a best power factor of 112 $\mu \mathrm{W} / \mathrm{cm}-\mathrm{K}^{2}$ at $300 \mathrm{~K}$ in $\mathrm{Si} / \mathrm{Ge} \mathrm{SL}$ structures, a factor of 5.6 better than current state-of-the-art bulk SiGe alloys and a factor of 8 better than a comparable SiGe alloy thin-film. The measurement of thermal conductivity (K) of $\mathrm{Si} / \mathrm{Ge} \mathrm{SL}$ structures indicates that short-period SL structures offer a significant reduction (by a factor of 2 to 4) in thermal conductivity compared to SiGe alloys. We observed a trend of decreasing $\mathrm{K}$ with a smaller SL period. Consideration for any potential anisotropy of $\mathrm{K}$ of Si/Ge SL structures, we estimate that the preliminary best ZT value is about a factor of five higher than state-of-the-art SiGe bulk alloys and about a factor ten better than comparable SiGe alloy thin-films. The results to date are promising, but tentative due to possible effects of the substrate and the developmental nature of measurements.

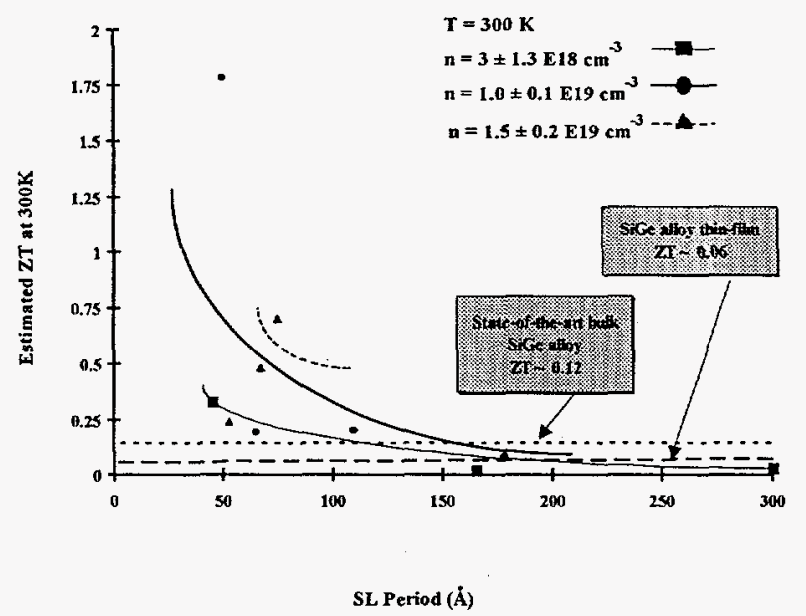

Figure 9 Variation of ZT at $300 \mathrm{~K}$ with SL period.

\section{References}

1. T.E. Whall and E.H. C. Parker, Proc. of 1st Euro. Conf. on Thermoelectrics, 51 (1987).

2. 1st National Thermogenic Cooler Conference, Sept. 17, 1992, Fort Belvoir, VA.

3. L.D. Hicks and M.S. Dresselhaus, Proceedings of the 1st National Thermogenic Cooler Workshop, Sept. 17, 1992, Fort Belvoir, VA.

4. T.C. Harman, Proceedings of at the 1st National Thermogenic Cooler Workshop, Sept. 17, 1992, Fort Belvoir, VA.

5. R. Venkatasubramanian, Proceedings of the 1st National Thermogenic Coler Workshop, Sept. 17, 1992, Fort Belvoir, VA.

6. N.B. Elsner et.al, Proc.of the 13th International Conf. on Thermoelectrics, AIP Conf. Proc., 316, 328 (1995).

7. T. Harman, Proc. of ARPA Thermoelectric Materials Workshop, Arlington, VA, Nov.1995.

8. R. Venkatsubramanian, Proc. of ARPA Thermoelectric Materials Workshop, Arlington, VA, Nov. 1995.

9. D.G. Cahill, M. Katiyar, and J.R. Abelson, Phys. Rev. B, 50, 6077 (1994). 